\author{
W. Blajer ${ }^{1}$ \\ Department of Mechanics. \\ Technical University of Radom. \\ ul. Malczewskiego 29. \\ 26.600 Radom, Poland \\ D. Bestle \\ W. Schiehlen \\ Institute B of Mechanics, \\ University of Stuttgart. \\ Pfaftenwaldring 9 . \\ 70550 Stuttgart 80 . Germany
}

\section{An Orthogonal Complement Matrix Formulation for Constrained Multibody Systems}

\begin{abstract}
A method is proposed for the automatic generation of an orthogonal complement matrix to the constraint matrix for the dynamic analysis of constrained multibody systems. The clue for this method lies in the determination of local constraint matrices and their orthogonal complements relative to the local reference frames of particular constrained points. These matrices are then transformed into the system's configuration space in order to form the final constraint matrix and its orthogonal complement. The avoidance of singularities in the formulation is discussed. The method is especially suited for the dynamic analysis of multibody systems with many constraints and/or closed-loops.
\end{abstract}

\section{Introduction}

Recently there has been increasing interest in efficient methods for the dynamic analysis of multibody systems subject to constraints, i.e., systems with closed loops or systems with specified motion. Usually, such systems lead to mixed differential-algebraic equations (DAEs) which are obtained by formulating the initial dynamical equations of the unconstrained (or open-loop) system, and then imposing the constraints. The corresponding dynamical equations are expressed in the initial (or dependent) coordinates and incorporate the constraint reactions by means of Lagrange multipliers. The direct integration of the equations needs the attachment of the constraint equations, and the problem is referred to the solution of a set of DAEs. Several methods for numerical treatment of such equations are available, e.g. [1]-[3], and many researchers prefer this approach due to its substantial simplicity and ease of derivation of the DAEs. Consequently, many general purpose multibody computer programs are based on this approach [4]. On the other hand, the numerical algorithms for solving the DAEs are commonly evaluated as computationally inefficient and unstable.

The difficulties in numerical treatment of DAE systems have stimulated the development of methods oriented at automatic elimination of constraint reactions from the analysis and resolving the governing equations into a more familiar form of purely differential equations. An additional advantage is the reduction of dimension of the problem. More or less manifestly and based on different principles of mechanics, the crux of the approach lies in the determination of a matrix being an orthogonal complement to the constraint matrix, see e.g. [5][12]. Since the ideal constraint reactions are, in principle, co-

'This work was done while the author was with the Institute B of Mechanics, University of Stuttgart, on behalf of the Alexander of Humboldt Foundation (Bonn-Bad Godesbers, Germany.)

Contributed by the Design Automation Committee for publication in the Journal of Mechanical Design. Manuscript received April 1991; revised Dec. 1992. Associate Technical Editor: O. A. Gabriele. linear with so-called constraint vectors contained in the constraint matrix, the multiplication of the initial (constraint reaction-containing) dynamical equations by the orthogonal complement matrix results in the reaction-free (or purely-kinetical) equations of motion.

The orthogonal complement matrix is determined either by an $a$ priori or literal choice of independent (or tangent) variables [5]-[7], [9], [11] or by numerical methods. In practice, the literal choice of the independent variables is often difficult and may lead to singularities. Therefore, a range of methods for the automatic generation of orthogonal complement matrices has grown rapidly in recent years. The coordinate partitioning (LU decomposition) method [7], the zero-eigenvalues theorem method [8], [13], the singular value decomposition method [14], [15], and the methods based on the Householder transformations [16], [17] and the Gram-Schmidt orthogonalization process [18], [19] are representative examples of methods of this type.

The objective of this paper is to present the development of a method for the automatic generation of an orthogonal complement matrix to the constraint matrix, suited for dynamic analysis of multibody systems with many constraints and/or closed-loops. The formulation of the method is based on the observation that the orthogonal and tangent directions to the constraint manifolds can easily be determined in local (often Cartesian) reference frames. The transformation of the local tangent directions into the system's configuration space is the key to the proposed method. The avoidance of singularities in the formulation is discussed, and some illustrative examples are included.

\section{Preliminary Definitions}

Consider an $(n-m)$-degree-of-freedom multibody system with closed-loops (internal constraints) and/or constraints due to contacting the environment (external constraints), see Fig. 1(a). Time-dependent constraints are also admissible for 
a)
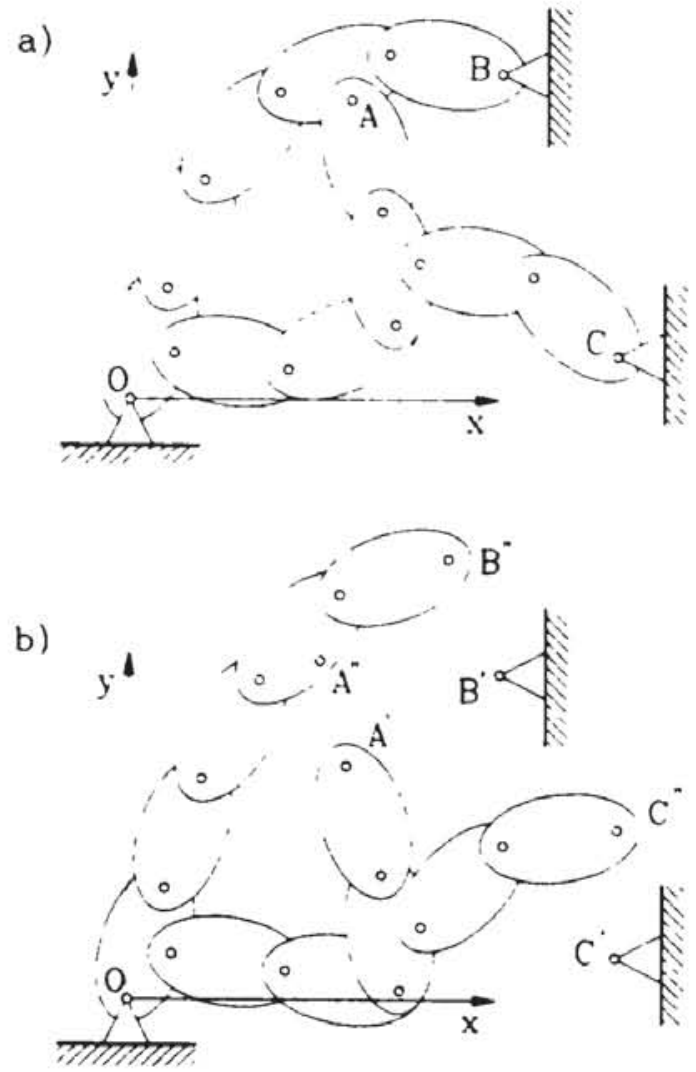

Fig. 1 A closed-loop/constrained and unconstrained multibody system

modelling specified motion requirements imposed on the system. In the first step, let us release the system from the $m$ constraints in order to build an $n$-degree-of-freedom open-loop (unconstrained) system, see Fig. 1(b). Note, that this can usually be done in many different ways.

The assumption of this paper is that the dynamics of the unconstrained system is determined by any method and, in fact, there is a range of methods for obtaining equations of motion for such systems. The corresponding dynamical equations can be written in the following form

$$
\mathbf{M}(\mathbf{q}, t) \ddot{\mathbf{q}}=\mathbf{h}(\dot{\mathbf{q}}, \mathbf{q}, t),
$$

where $M$ is the $n \times n$ symmetric positive-definite mass matrix; $q=\left[q_{1}, \ldots, q_{n}\right]^{T}$ is the column matrix representation of the unconstrained system coordinates; $\mathbf{h}=\left[h_{1}, \ldots, h_{n}\right]^{T}$ represents the external, centrifugal, and Coriolis forces on the system; and $t$ is the time.

In order to retrieve the behavior of the closed-loop/constrained system, the removed constraints must be imposed on the unconstrained system. Assume that there are $p$ constrained points with $m_{i}$ constraints at the $i$ th constrained point $\left(m_{1}+\ldots+m_{p}=m\right)$, which constrain the relative position ( $p o-$ sition implies both position and orientation) of the contacting bodies (internal constraints) or the position of the bodies in contact with the environment (external constraints). Let us introduce the constraint equations by means of the ith constrained point local coordinates $x_{i}=\left[x_{i 1}, \ldots, x_{i n}\right]^{T}$

$$
\mathbf{f}_{i}\left(\mathbf{x}_{i}, t\right)=\mathbf{0},
$$

where $\boldsymbol{f}_{i}=\left[\hat{f}_{i 1}, \ldots, \hat{f}_{i m_{m}}\right]^{T}, m_{i} \leq n_{i}$. The superscript ( $\left.{ }^{\circ}\right)$ denotes that the corresponding function is expressed in the local coordinates $\mathbf{x}_{i}$ which describe the relative position of the contacting bodies (internal constraints) or the position of the body in contact with the environment (external constraints), hence $n_{1} \leq 6$ (for the internal constraint at point $A$ in Fig. $1, x_{i}$ is assumed to be equal to $\mathbf{x}_{A}=\mathbf{x}_{A^{\prime \prime}}{ }^{\prime \prime} x_{A^{\prime}}$, where $x_{A^{\prime \prime}}$ and $x_{A^{\prime}}$ are the positions of points $A^{\prime \prime}$ and $A^{\prime}$ relative the inertial frame). From the point of view of efficiency of the reported method it is essential to choose a minimal number of local coordinates $\mathbf{x}_{i}$ for defining the constraints (2), if possible $n_{t}=m_{l}$ (the problem will be discussed more precisely later on).

The interdependence between the local coordinates $\mathbf{x}_{4}$ and the system's initial coordinates $\mathbf{q}$ can be written as

$$
\mathbf{x}_{\boldsymbol{i}}=\mathbf{g}_{\mathbf{i}}(\mathbf{q}) \text {, }
$$

where $g_{\text {, }}$ contains at least twice differentiable functions. Using this, the constraints (2) can be expressed in $q$ and $t$, i.e., $\mathbf{f}_{i}(\mathbf{q}, t)=\boldsymbol{f}_{i}(\mathbf{g},(\mathbf{q}), t)=\mathbf{0}(i=1, \ldots, p)$. Then, defining the total vector of constraints on the system as $\mathbf{f}=\left[\mathbf{f}_{1}^{T}, \ldots, \mathbf{f}_{\rho}^{T}\right]^{T}$, the initial (constraint reaction-containing) governing equations of the motion of the system are:

$$
\begin{gathered}
\mathbf{M} \ddot{\mathbf{q}}=\mathbf{h}+\mathbf{C}^{\boldsymbol{T}} \boldsymbol{\lambda}, \\
\mathbf{C} \ddot{\mathbf{q}}+\mathbf{c}_{0}=\mathbf{0},
\end{gathered}
$$

where $\mathbf{C}(\mathbf{q}, t)=\partial \boldsymbol{t} / \partial \mathbf{q}=\left[\mathbf{C}_{1}^{T}, \ldots, \mathbf{C}_{p}^{T}\right]^{T}$ is the $m \times n$ constraint matrix, and $C_{1}=\partial f_{1} / \partial q$ refers to the $i$ th constrained point; $\lambda=\left[\lambda_{1}^{T}, \ldots, \lambda_{p}^{T}\right]^{T}$ contains $m$ Lagrange multipliers, $\lambda_{1}=\left[\lambda_{t 1}\right.$, $\left.\ldots, \lambda_{t m}\right]^{T}$; and $\mathbf{c}_{0}(\mathbf{q}, t)=\left[\mathbf{c}_{01}, \ldots, \mathbf{c}_{0 p}\right]^{T}$ is the $m$-dimensional column matrix, $\mathbf{c}_{0_{t}}=\left(\partial \mathbf{f}_{t} / \partial \mathbf{q}\right)^{\prime} \dot{\mathbf{q}}+\left(\partial \mathbf{f}_{t} / \partial t\right)^{\circ}$. One can easily deduce that (5) expresses the constraint equations transformed to the second-order kinematical form, and the initial conditions $\mathbf{f}\left(\mathbf{q}_{0}, 0\right)=\mathbf{0}$ and $\mathbf{f}\left(\dot{q}_{0}, \mathbf{q}_{0}, 0\right)=\mathbf{0}$ have to be satisfied .

The governing Eqs. (4) and (5) form a set of DAEs, and the numerical treatment of such equations may be computationally inefficient. Thus, methods aimed at automatic elimination of the constraining forces $\mathbf{C}^{T} \lambda$ from the analysis, and consequently reducing the dimension of the problem and transforming the resultant equations to the form of purely differential equations, are often introduced. The clue to these methods, mentioned in Section 1, consists in the left-sided premultiplication of the dynamical Eqs. (4) by an $(n-m) \times n$ orthogonal complement matrix $\mathbf{D}(\mathbf{q}, t)$ such that $\mathbf{D} \mathbf{C}^{t}=\mathbf{0}$, which represents the vanishing virtual work of the constraint forces. As a result, the dynamical equations transform to the following form

$$
\mathbf{D M} \ddot{\mathbf{q}}=\mathbf{D h} .
$$

These reaction-free dynamical equations, mixed with the constraint Eqs. (5), form the governing differential equations of the motion.

As the constraint matrix $\mathbf{C}$ can be obtained by mathematical manipulations when (2) and (3) are defined, the determination of D is usually not so evident. For small systems, D can be often found by inspection (see, e.g., [6], [12], [20]); for large systems the literal determination of $\mathbf{D}$ is much more complicated. Hence, in applications $\mathbf{D}$ is usually determined with the use of numerical methods, [7], [13]-[19]. In this paper another method for the automatic generation of the orthogonal complement matrix is proposed. This method is conceptually simple and assures avoidance of singularities in the analysis. Moreover it gives an interesting insight into the problem of dynamic analysis of constrained systems, and may have a tutorial value as well.

Of critical importance for further formulation is the observation that the columns of $\mathbf{C}^{T}$ and $\mathbf{D}^{T}$ are, respectively, the covariant representations of the constraint vectors spanning the constrained subspace, and the contravariant representations of vectors spanning the tangent (null) subspace in the system's configuration space treated as an $n$-dimensional Riemannian space. The same concerns the constraint matrices and their orthogonal complements in the local reference frames ( $n_{r}$-dimensional Riemannian spaces). This yields different formulae for the transformation of the local constraint matrices and the local orthogonal complement matrices into the system's configuration space. These aspects are described in detail in [12]. The present paper has been deliberately written in the simplest possible, standard, engineering notation. Only in some places and when it is indispensable, the reader is referred to [12] for the mathematical background. 


\section{Solution}

The reported method is based on the observation that $\hat{\mathbf{D}}$, can be easily found as an orthogonal complement on $\hat{\mathbf{C}}_{t}$ in the $n_{i}$-dimensional $\left(n_{i} \leq 6\right)$ subspace referred to the $i$ th constrained point $(i=1, \ldots, p)$. The transformation of the local $\hat{\mathbf{D}}_{i}$ matrices into the configuration space is, generally speaking, the way to build the matrix D used in (6).

As stated in (2), the constraint equations are expressed in local coordinates $\mathbf{x}_{i}$. Defining

$$
\hat{\mathbf{C}}_{i}=\partial \hat{\mathbf{f}}_{i} / \partial \mathbf{x}_{i},
$$

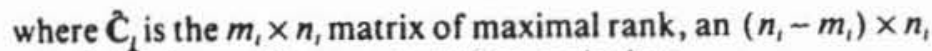

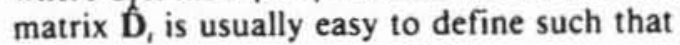

$$
\hat{\mathbf{D}}, \hat{C}_{t}^{T}=\mathbf{0} \text {. }
$$

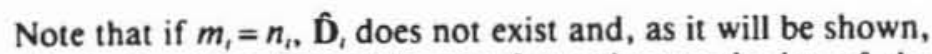
this is the most favorable case from the standpoint of the efficiency of the method.

Let us build the global vector of the local coordinates, $\mathbf{x}=\left[\mathbf{x}_{1}^{T}\right.$, $\left.\ldots, \mathbf{x}_{p}^{T}\right]^{T}$, and according to (3) introduce the relationship

$$
x=\left[\begin{array}{c}
x_{t} \\
\vdots \\
x_{p}
\end{array}\right]=g(q)=\left[\begin{array}{c}
g_{i}(q) \\
\vdots \\
g_{\rho}(q)
\end{array}\right] .
$$

Denoting the dimension of $\mathrm{x}$ by $k\left(k=n_{1}+\ldots+n_{p}\right)$, the following $k \times n$ Jacobian matrix can be defined

$$
\mathbf{J}(\mathbf{q})=\partial \mathbf{x} / \partial \mathbf{q}=\left[\begin{array}{c}
\partial \mathbf{g}_{1} / \partial \mathbf{q} \\
\vdots \\
\partial \mathbf{g}_{\rho} / \partial \mathbf{p}
\end{array}\right]
$$

which is the transformation matrix relating $\dot{\mathbf{x}}$ and $\dot{\mathbf{q}}, \dot{\mathbf{x}}=\mathbf{J} \dot{\mathbf{q}}$.

Using the above definitions, the global $k \times m$ matrix $\hat{C}^{T}$ and the global $k \times(n-m)$ matrix $\hat{\mathbf{D}}^{T}$ are:

$$
\begin{aligned}
\hat{\mathbf{C}}^{T} & =\left[\begin{array}{cccc}
\mathbf{C}_{1}^{T} & \mathbf{0} & \cdots & \mathbf{0} \\
\mathbf{0} & \hat{\mathbf{C}}_{2}^{T} & \cdots & \mathbf{0} \\
\vdots & \vdots & & \vdots \\
\mathbf{0} & \mathbf{0} & \cdots & \hat{\mathbf{C}}_{p}^{T}
\end{array}\right], \\
\hat{\mathbf{b}}^{T} & =\left[\begin{array}{cccc}
\mathbf{\mathbf { D }}_{1}^{T} & \mathbf{0} & \cdots & \mathbf{0} \\
\mathbf{0} & \mathbf{\mathbf { D }}_{2}^{T} & \cdots & \mathbf{0} \\
\vdots & \vdots & & \vdots \\
\mathbf{0} & \mathbf{0} & \cdots & \mathbf{D}_{p}^{T}
\end{array}\right],
\end{aligned}
$$

where $\mathbf{0}$ denotes the null matrix of proper dimension.

Since the columns of $\hat{\mathbf{C}}^{T}$ are the covariant components of the constraint vectors in the local reference frames, the covariant representation of the vectors in the system's configuration space can be obtained by using the following transformation formula (refer to [12] for the background)

$$
\mathbf{C}^{T}=\mathbf{J}^{T} \mathbf{C}^{T} \text {. }
$$

As said previously, the columns of $\hat{\mathbf{D}}^{T}$ are contravariant representations of vectors spanning the tangent directions in the local reference frames. Hence, following the formulation given in [12], the transformation formula reads as $\mathbf{D}^{T}=\mathbf{J}^{-1} \hat{\mathbf{D}}^{T}$, and demands $k=n$ and $\operatorname{rank}(\mathrm{J})=n$. For a general case, however, $k \leq n$, and for $k<n$ the following approach should be undertaken.

Let us append the $k$ local coordinates $\mathrm{x}$ by $\boldsymbol{n}-\boldsymbol{k}$ complementary coordinates $\mathbf{y}$, and define the full dimension extended local coordinates $\mathbf{x}^{\prime}$

$$
x^{\prime}=\left[\begin{array}{l}
x \\
y
\end{array}\right]=g^{\prime}(q)=\left[\begin{array}{l}
g(q) \\
\gamma(q)
\end{array}\right],
$$

$$
\mathbf{J}^{\prime}=\partial \mathbf{g}^{\prime} / \partial \mathbf{q}=\left[\begin{array}{l}
\partial \mathbf{g} / \partial \mathbf{q} \\
\partial \boldsymbol{\gamma} / \partial \mathbf{q}
\end{array}\right]=\left[\begin{array}{l}
\mathbf{J} \\
\mathbf{G}
\end{array}\right] \text {, }
$$

is of maximal rank (note that $\dot{\mathbf{x}}^{\prime}=\mathrm{J}^{\prime} \dot{\mathbf{q}}$ ).

Irrespective of the choice of $\mathbf{y}$, the matrices $\mathbf{C}^{\prime T}$ and $\hat{\mathbf{D}}^{\prime T}$, referred to the extended local coordinates $\mathbf{x}^{\prime}$, can be defined as

$$
\begin{array}{r}
\hat{\mathbf{C}}^{\prime T}=\left[\begin{array}{c}
\hat{\mathbf{C}}^{T} \\
k \times m \\
\mathbf{0} \\
(n-k) \times m
\end{array}\right], \\
\hat{\mathbf{D}}^{\prime T}=\left[\begin{array}{cc}
\hat{\mathbf{D}}^{T} & \mathbf{0} \\
k \times(k-m) & k \times(n-k) \\
\mathbf{0} & \mathbf{I} \\
(n-k) \times(k-m) & (n-k) \times(n-k)
\end{array}\right],
\end{array}
$$

where $\mathbf{0}$ and $\mathbf{I}$ denote the zero and unit matrices with the dimensions indicated. Note that the ranks of $\hat{C}^{\prime T}$ and $\hat{\mathbf{D}}^{\prime T}$ are maximal, and equal $m$ and $n-m$, respectively, if only the ranks of $\hat{C}^{T}$ and $\hat{\mathbf{D}}^{T}$ are maximal.

Now, in order to build $\mathbf{C}$ and $\mathbf{D}$, the following formulae are available:

$$
\begin{gathered}
\mathbf{C}^{T}=\mathbf{J}^{\prime}{ }^{T} \hat{\mathbf{C}}^{\prime T}=\mathbf{J}^{T} \hat{\mathbf{C}}^{T}, \\
\mathbf{D}^{T}=\left(\mathbf{J}^{\prime}\right)^{-1} \hat{\mathbf{D}}^{\prime T},
\end{gathered}
$$

As said, $\hat{\mathbf{C}}^{\prime T}$ and $\hat{\mathbf{D}}^{\prime T}$ can be built irrespectively of the choice of $\mathbf{y}$. A reasonable choice for $\mathbf{y}$ is to set them as appropriate $n-k$ elements from q. The $(n-k) \times n$ matrix $\mathbf{G}$ defined in Eq. (14) will then contain only one nonzero (equal to 1) entry in each row. Exploiting this, the matrices $\mathbf{J}$ and $\mathbf{G}$ can symbolically be written as $\tilde{\mathbf{J}}=\left[\begin{array}{ll}\mathbf{J}_{1} & \mathbf{J}_{2}\end{array}\right]$ and $\tilde{\mathbf{G}}=\left[\begin{array}{ll}\mathbf{I} & \mathbf{0}\end{array}\right]$, where $\mathbf{I}$ denotes the $(n-k) \times(n-k)$ identity matrix, and the $k \times k$ matrix $J_{2}$ must be invertible $\left(\operatorname{det}\left(\mathbf{J}_{2}\right) \neq 0\right)$ in order to assure invertibility of $\mathbf{J}^{\prime}$. The matrix $\left(\mathbf{J}^{\prime}\right)^{-1}$ used in Eq. (17) can then be represented symbolically as

$$
\left(\tilde{\mathbf{J}}^{\prime}\right)^{-1}=\left[\begin{array}{cc}
0 & \mathbf{I} \\
(n-k) \times k & (n-k) \times(n-k) \\
\mathbf{J}_{2}^{-1} & -\mathbf{J}_{2}^{-1} \mathbf{J}_{1} \\
k \times k & k \times(n-k)
\end{array}\right] .
$$

The symbolic representation of Eq. (18) means that the rows of $\left(\tilde{J}^{\prime}\right)^{-1}$ as compared with $\left(\mathbf{J}^{\prime}\right)^{-1}$ are in the same setting as the columns of the symbolic partition $\tilde{\mathbf{J}}=\left[\begin{array}{ll}\mathbf{J}_{1} & \mathbf{J}_{2}\end{array}\right]$ as compared with $\mathbf{J}$ used in Eq. (14). These processes can easily be automatized in computations.

Summarizing, the following algorithm of the reported method can be proposed.

\section{Algorithm}

1. Release the system from the constraints, and derive the dynamical Eqs. (1) of the unconstrained system;

$2^{\circ}$ Formulate the constraint Eqs. (2) expressed in the local coordinates of the $i$ th constrained point $(i=1, \ldots, p)$;

$3^{\circ}$ Introduce the interdependences (3) between the $x_{i}$ and the $\mathbf{q}$ coordinates, and formulate the Jacobian matrix $\mathbf{J}$ according to (10);

$4^{\circ}$ Formulate $\hat{C}_{i}$ and $\hat{\mathbf{D}}_{i}(i=1, \ldots, p)$ according to $(7)$ and (8), respectively. If $m_{i}=n_{i}, \hat{\mathbf{D}}_{i}$ does not exist;

$5^{\circ}$ Build matrices $\mathbf{C}^{T}$ and $\hat{\mathbf{D}}^{T}$, and then $\hat{C}^{\prime T}$ and $\hat{\mathbf{D}}^{\prime T}$ according to (11) and (15), respectively;

$6^{\circ}$ Partition $\mathbf{J}$ symbolically to $\mathbf{J}=\left[\begin{array}{ll}\mathbf{J}_{1} & \mathbf{J}_{2}\end{array}\right]$ so that the $k \times k$ matrix $\mathbf{J}_{2}$ is invertible and calculate $\left(\mathbf{J}^{\prime}\right)^{-1}$ using the symbolic relation (18)

$7^{\circ}$ Determine $\mathbf{D}$ (and C, if needed) according to (17) and (16). 


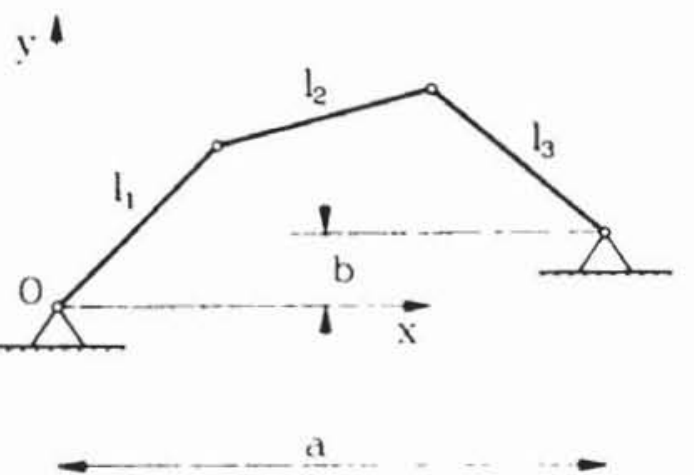

Fig. 2 The constrained system of Exampie 1
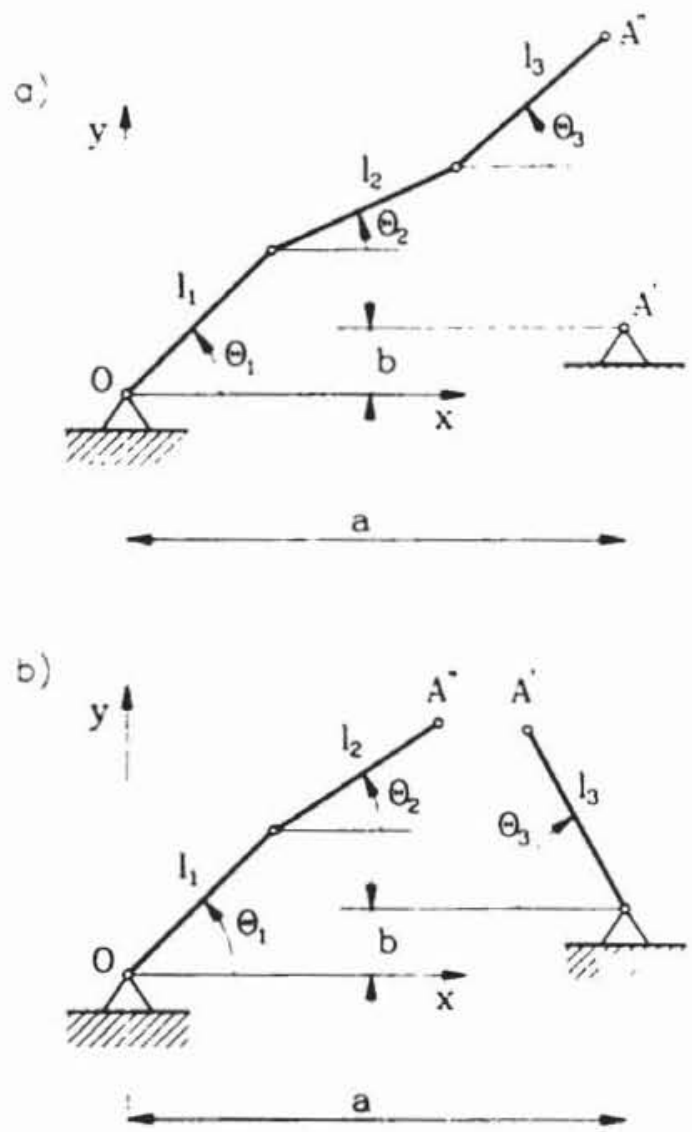

Flg. 3 The unconstrained systems of Example 1

\section{Applications}

Example 1. Let us consider first the four-bar linkage shown in Fig. 2. Two possible ways of constructing the unconstrained system will be discussed, see Fig. 3. Denoting $q=\left[\theta_{1}, \theta_{2}, \theta_{3}\right]^{T}$, the dynamical equations of each of the unconstrained systems can be derived in form (1). For brevity, the equations will not be reported here. (In fact, the equations for Case b are simpler then the equations for Case a. Hence, cutting closed-loops in the middle can be recommended).

Introducing the local coordinates $x_{A}$ and $y_{A}$ as relative position coordinates of $A^{\prime \prime}$ and $A^{\prime}, x_{A}=x_{A^{*}}-x_{A^{\prime}}$, and $y_{A}=y_{A}{ }^{*}-y_{A}$, the constraint equations for both Cases are

$$
j_{1}=x_{A}=0, \quad j_{2}=y_{A}=0 \text {. }
$$

The local constraint matrix Ĉ, corresponding to (11), is (for both Cases)

$$
\hat{C}=\left[\begin{array}{ll}
1 & 0 \\
0 & 1
\end{array}\right] \text {, }
$$

and the local $\mathbf{D}$ matrix does not exist because of $m=k=2$.
Thus, the extended dimension matrices ' $\mathbf{C}^{\prime}$ and $\hat{\mathbf{b}}^{\prime}$ are:

$$
\mathbf{C}^{\prime} T=\left[\begin{array}{ll}
1 & 0 \\
0 & 1 \\
0 & 0
\end{array}\right], \quad \mathbf{D}^{\prime T}=\left[\begin{array}{l}
0 \\
0 \\
1
\end{array}\right],
$$

From kinematics we find:

$$
\begin{aligned}
& \mathbf{x}_{c}=\left[\begin{array}{l}
x_{A} \\
y_{A}
\end{array}\right]_{a}=\left[\begin{array}{l}
l_{1} \cos \theta_{1}+l_{2} \cos \theta_{2}+l_{3} \cos \theta_{3}-a \\
l_{1} \sin \theta_{1}+l_{2} \sin \theta_{2}+l_{3} \sin \theta_{3}-b
\end{array}\right] . \\
& \mathbf{x}_{b}=\left[\begin{array}{l}
x_{A} \\
y_{A}
\end{array}\right]_{b}=\left[\begin{array}{l}
l_{1} \cos \theta_{1}+l_{2} \cos \theta_{2}-a+l_{3} \cos \theta_{3} \\
l_{1} \sin \theta_{1}+l_{2} \sin \theta_{2}-b-l_{3} \sin \theta_{3}
\end{array}\right] .
\end{aligned}
$$

respectively, and the Jacobians defined in (10) are:

$$
\begin{aligned}
& \mathbf{J}_{a}=\left[\begin{array}{ccc}
-l_{1} \sin \theta_{1} & -l_{2} \sin \theta_{2} & -l_{3} \sin \theta_{3} \\
l_{1} \cos \theta_{1} & l_{2} \cos \theta_{2} & l_{3} \cos \theta_{3}
\end{array}\right] . \\
& \mathbf{J}_{b}=\left[\begin{array}{ccc}
-l_{1} \sin \theta_{1} & -l_{2} \sin \theta_{2} & -l_{3} \sin \theta_{3} \\
l_{1} \cos \theta_{1} & l_{2} \cos \theta_{2} & -l_{3} \cos \theta_{3}
\end{array}\right] .
\end{aligned}
$$

The extended Jacobian may be chosen as

$$
\mathbf{J}_{1}^{\prime}=\left[\begin{array}{ccc} 
& \mathbf{J}_{1} \\
\cdots & \cdots & \cdots \\
0 & i & 0
\end{array}\right], \quad i=a, b,
$$

which corresponds to the extended local coordinates $\mathbf{x}^{\prime}=\left[x_{A}, y_{A}, \theta_{2}\right]^{\dagger}$. (Note that " 1 " in (24) can change its column position according to the choice of $\theta_{1}, i=1,2$, or 3 , in the extended local coordinate vector $\mathbf{x}^{\prime}$ ). For this particular position of "1," the matrix D can be found from (17) as

$$
\begin{aligned}
& \mathbf{D}_{a}^{T}=\left[\begin{array}{c}
-\frac{l_{2} \sin \left(\theta_{3}-\theta_{2}\right)}{l_{1} \sin \left(\theta_{3}-\theta_{1}\right)} \\
1 \\
-\frac{l_{2} \sin \left(\theta_{2}-\theta_{1}\right)}{l_{3} \sin \left(\theta_{3}-\theta_{1}\right)}
\end{array}\right], \\
& \mathbf{D}_{b}^{T}=\left[\begin{array}{c}
-\frac{l_{2} \sin \left(\theta_{3}+\theta_{2}\right)}{l_{1} \sin \left(\theta_{3}+\theta_{1}\right)} \\
1 \\
-\frac{l_{2} \sin \left(\theta_{2}-\theta_{1}\right)}{l_{3} \sin \left(\theta_{3}+\theta_{1}\right)}
\end{array}\right] .
\end{aligned}
$$

Regarding the problem of singularity of $\mathbf{J}^{\prime}$, for both Cases $\operatorname{rank}(J)=\max =2$ is assured unless all three links are parallel. Leaving this case out of the analysis, the local singularity may occur when any two links are parallel. When the ith and the $j$ th links are parallel, $i j=1,2,3$, the $2 \times 2$ submatrix formed by the $i$ th and $j$ th columns of $\mathbf{J}$ is singular; the determinant of the submatrix equals zero. Hence " $I$ " in $\mathbf{J}$ ' must appear either in the ith or jth column position in order to assure the maximal rank and invertibility of $\mathbf{J}^{\prime}$. The second-column position of " 1 " in (24) is correct for $\sin \left(\theta_{3}-\theta_{1}\right) \neq 0$ (Case a) and $\sin \left(\theta_{3}+\theta_{1}\right) \neq 0$ (Case b). Otherwise, "1" should be placed in the first- or third-column position.

Example 2. Now, let us modify the example to the problem shown in Fig. 4. Building the unconstrained system as in Fig. $3(a)$, the constraint equation is

$$
f_{a}=y_{1}-\left(x_{1}-\frac{c}{\sin \alpha}\right) \tan \alpha=0,
$$

where $x_{A}$ and $y_{A}$ are the position coordinates of the point $A$ in the $x y$-reference frame. On the other hand, the constraint expressed in the $x_{v} y_{1}$-reference frame takes a more simple form, i.e.,

$$
j_{b}=x_{1 A}-c=0 .
$$

As stated in Section 3, the description of the constraints on 


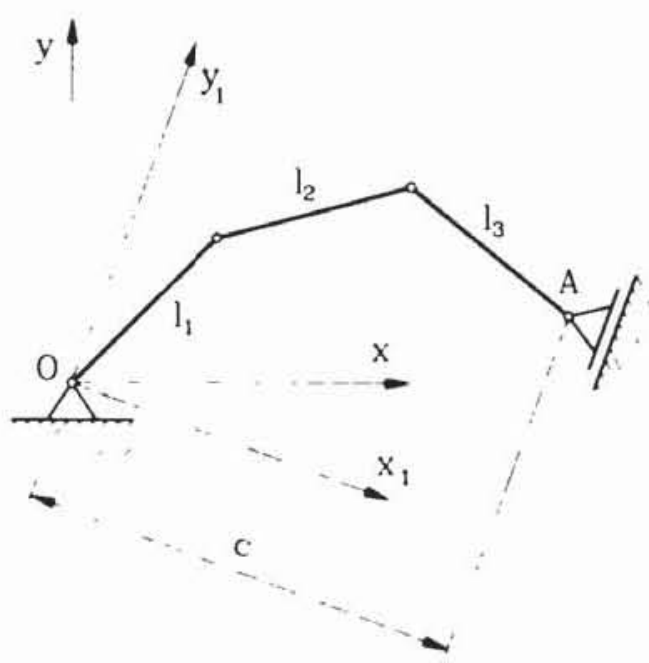

Fig. 4 Examplo 2 illustration

the system by a possible minimal set of local coordinates gives advantages in the analysis. These advantages will be illustrated here by comparing the two cases of the constraint formulation: Case a, (26a), where two local coordinates $x_{A}$ and $y_{A}$ are introduced; and Case b, $(26 b)$, where only one local coordinate $x_{1 A}$ is introduced. The mathematical formulations for the two cases are:

$$
\begin{aligned}
& \hat{\mathbf{C}}_{a}^{T}=\left[\begin{array}{c}
-\tan \alpha \\
1
\end{array}\right], \quad \hat{\mathbf{C}}_{b}^{T}=[1], \\
& \mathbf{J}_{a}=\left[\begin{array}{ccc}
-l_{1} \sin \theta_{1} & -l_{2} \sin \theta_{2} & -l_{3} \sin \theta_{3} \\
l_{1} \cos \theta_{1} & l_{2} \cos \theta_{2} & l_{3} \cos \theta_{3}
\end{array}\right] \text {, } \\
& \mathbf{J}_{b}=\left[-l_{1} \cos \left(\theta_{1}-\alpha\right)-l_{2} \cos \left(\theta_{2}-\alpha\right)-l_{3} \cos \left(\theta_{3}-\alpha\right)\right] \text {. } \\
& \hat{\mathbf{D}}_{\alpha}^{T}=\left[\begin{array}{c}
1 \\
\tan \alpha
\end{array}\right], \quad \hat{\mathbf{D}}_{b} \text { does not exist, } \\
& \hat{\mathbf{C}}_{a}^{\prime T}=\left[\begin{array}{c}
-\tan \alpha \\
1 \\
0
\end{array}\right], \quad \mathbf{C}_{b}^{\prime}{ }^{T}=\left[\begin{array}{l}
1 \\
0 \\
0
\end{array}\right], \\
& \hat{\mathbf{b}}_{a}^{\prime}=\left[\begin{array}{cc}
1 & 0 \\
\tan \alpha & 0 \\
0 & 1
\end{array}\right], \quad \hat{\mathbf{D}}_{b}^{\prime T}=\left[\begin{array}{ll}
0 & 0 \\
1 & 0 \\
0 & 1
\end{array}\right],
\end{aligned}
$$

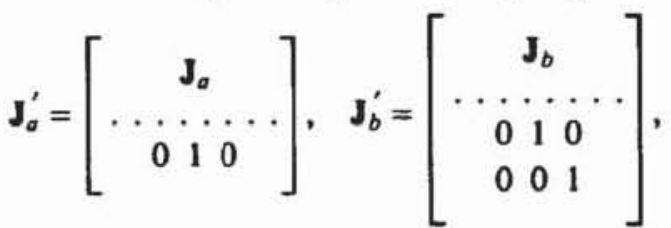

$$
\begin{aligned}
& \mathbf{D}_{a}=\left[\begin{array}{ccc}
\frac{\cos \theta_{3}+\sin \theta_{3} \tan \alpha}{l_{1} \sin \left(\theta_{3}-\theta_{1}\right)} & 0 & -\frac{\cos \theta_{1}+\sin \theta_{1} \tan \alpha}{l_{3} \sin \left(\theta_{3}-\theta_{1}\right)} \\
-\frac{l_{2} \sin \left(\theta_{3}-\theta_{2}\right)}{l_{1} \sin \left(\theta_{3}-\theta_{1}\right)} & 1 & -\frac{l_{2} \sin \left(\theta_{2}-\theta_{1}\right)}{l_{3} \sin \left(\theta_{3}-\theta_{1}\right)}
\end{array}\right] \text {, } \\
& \mathbf{D}_{b}=\left[\begin{array}{ccc}
-\frac{l_{2} \cos \left(\theta_{2}-\alpha\right)}{l_{1} \cos \left(\theta_{1}-\alpha\right)} & 1 & 0 \\
\frac{l_{3} \cos \left(\theta_{3}-\alpha\right)}{l_{1} \cos \left(\theta_{1}-\alpha\right)} & 0 & 1
\end{array}\right] \text {, }
\end{aligned}
$$

respectively.

\section{Discussion}

The reported method seems to be conceptually simple and easy to apply. Steps $1^{\circ}-5^{\circ}$ of the algorithm described in Section 3 should be completed in the stage of physical and mathematical modelling of the problem, and the numerical treatment is needed only in Steps $6^{\circ}$ and $7^{\circ}$ to be executed repeatedly. It is worth noting that Step $6^{\circ}$, which can be easily automatized in computations, may be aimed not only at avoiding the singularities but also at building the best conditioned matrix $\mathbf{J}^{\prime}$ as well, which may be of importance in calculations. Note also that changes in formulating $\mathbf{J}^{\prime}$, and through it in determining D, do not affect the integration process of (6) and (5) carried out in q. This is still valid for the analysis of constraint addition/deletion problems since the total dimension of $\mathbf{D}$ and C is constantly $n$. The utility of the method has also been proved for the symbolic derivation of the orthogonal complement matrices.

The numerical treatment of the $n-m$ dynamical Eqs. (6) requires that they must be combined with $m$ constraint equations in the second-order kinematical form (5) (note that the matrix $\left[\mathbf{M D}^{T}, \mathbf{C}^{T}\right]$ is of maximal rank in principle). In order to avoid the constraint violation due to the numerical errors of integration, Baumgarte's constraint stabilization method may be applied [21, 22]. In the meaning of this method, the dynamical equations (6) should be mixed with

$$
\mathbf{C} \ddot{\mathbf{q}}+\mathbf{c}_{0}+\mathbf{K}_{v} \dot{\mathbf{f}}+\mathbf{K}_{p} \mathbf{f}=\mathbf{0},
$$

where $\mathbf{K}_{v}$ and $\mathbf{K}_{p}$ are diagonal matrices of appropriately chosen gain values. However, the well-known shortcomings of the numerically erroneous Baumgart's method cannot be avoided, for more details see [22].

Beside the formulation of the constraint reaction-free dynamical Eqs. (6), the reactions of the rejected constraints can be retrieved. Namely, the Lagrange multipliers, introduced in (4), can be found as

$$
\lambda=-\left(\mathbf{C M}^{-1} \mathbf{C}^{T}\right)^{-1}\left(\mathbf{c}_{0}+\mathbf{C M}^{-1} \mathbf{h}\right)=\lambda(\dot{\mathbf{q}}, \mathbf{q}, t),
$$

and the $j$ th $(j=1, \ldots, m)$ constraint reaction is

$$
\mathbf{r}_{j}=\mathbf{c}_{j} \lambda_{j}=\mathbf{r}_{j}(\dot{\mathbf{q}}, \mathbf{q}, t),
$$

where $\mathbf{c}_{j}$ is the $j$ th column of $\mathbf{C}^{T}$. Note that the vector of the $j$ th constraint reaction is represented in (36) by covariant components in the system's configuration space. Hence, neither $\boldsymbol{\lambda}_{j}$ nor $\operatorname{sqrt}\left(r_{11}^{2}+\ldots+r_{j n}^{2}\right)$ is, in general, the constraint reaction value in the physical sense; the configuration space may not, in general, be a physical space. In order to retrieve the physical meaning of the constraint reactions, they should be retransformed to the local reference frames, where the constraint reactions can be easily interpreted. The transformation is as follows (refer to [12])

$$
\left[\begin{array}{l}
\mathbf{P}_{j} \\
\mathbf{0}
\end{array}\right]=\left(\mathbf{J}^{\prime}\right)^{-1} \mathbf{r}_{j}=\left[\begin{array}{c}
\mathbf{c}_{,} \lambda_{j} \\
\mathbf{0}
\end{array}\right],
$$

where $\boldsymbol{c}_{j}$ is the $j$ th column of $\hat{\mathbf{C}}^{\boldsymbol{T}}$ defined in (11), and $\hat{\mathbf{r}}_{j}$ is a column matrix of dimension $k$. In other words, (37) projects the constraint reactions $\mathbf{r}_{j}$, defined in the system's configuration space, into the space defined by $\mathbf{x}$, and each of the constraint reactions is represented only in a particular local reference frame of $\mathbf{x}_{i}$ in which the corresponding constraint is defined; see the structure of $\hat{C}^{T}$ defined in (11).

From (4) and (35) it follows immediately that [23]

$$
\mathbf{M} \ddot{\mathbf{q}}=\mathbf{h}-\mathbf{C}^{T}\left(\mathbf{C M}^{-1} \mathbf{C}^{T}\right)^{-1}\left(\mathbf{c}_{0}+\mathbf{C M}^{-1} \mathbf{h}\right) \text {. }
$$

This system of equations has also dimension $n$ as Eqs. (5) and (6), and the evaluation of matrix $\mathbf{D}$ is not required. In order to avoid the numerical instability and constraint violation due to numerical errors of integration, as previously, Baumgart's constraint stabilization method may be applied, i.e., (38) may be replaced by

$$
\mathbf{M} \ddot{\mathbf{q}}=\mathbf{h}-\mathbf{C}^{T}\left(\mathbf{C M}^{-1} \mathbf{C}^{T}\right)^{-1}\left(c_{0}+\mathbf{C M}^{-1} \mathbf{h}+\mathbf{K}_{v} \dot{\mathbf{f}}+\mathbf{K}_{p} \mathbf{f}\right),
$$
and the shortcomings of Baumgart's method are still valid. 
The computational efficiency of both the approaches, i.e., by the use of the orthogonal complement matrix and by the direct elimination of $\lambda$ as stated in (38), is an open question. In [24], however, the two methods are examined in detail, and the advantages of the first method are emphasized. Moreover, the concept of the orthogonal complement matrix is introduced and used in many other papers concerning the dynamic analysis of constrained systems, e.g., in [4]-[20], which prove the utility of the approach. This work contributes to the methods for the generation of orthogonal complement matrices.

\section{References}

I Gear, C. W., and Petzold. L. R., 1984, "ODE Methods for the Solution of Differentual/Algebrac Systems," SIAM Journal on Numerical A nalysis, Vol 21, pp. 716-728.

2 Gear, C. W., Leimkuhler, B., and Gupa, G. K , 1985. "Automatic in. tegration of Euler-Lagrange Equations with Constraints," Journal of Computational and Applied Mathematics. Vol 12/13. pp 77-90

3 Fuhrer, C., and Leimkuhler, B. J., 1991, "Numerical Solution of Dif. ferential-Algebraic Equations for Constrauned Mechanical Systems," Numer. sche Mathematik, Vol. 59, pp. 55-69.

4 Nikravesh, P. E., 1988, Computer-Aided A nalysis of Mechanical Sysiems. Prince-Hall, Englewood Chifs, NJ.

5 Kane, T. R., and Levinson. D A., 1980, "Formulation of Equations of Motion for Complex Spacecraft." AIAA Journal of Gutance and Control, Vol. 3, pp. 99-112.

6 Hemami, H., and Wermer, F C.. 1981. "Modelling of Nonholonomic Dynamic Systems with Applications." ASME Journal of Applied Mechanics. Vol. 48, pp. 177-182.

7 Wehage, R. A., and Haug, E. J., 1982, "Generalized Cootdinate Partı. toning for Dimension Reduction in Analysis of Constrained Dynamic Systems," ASME Jouranal of Mechanical Design, Vol 104. PP 247-255.

8 Kamman, J. W., and Huston. R. L., 1984, "Dynamics of Constrained Multibody Systems," ASME Joumal of Applied Mechanics, Vol. 51. pp 899903.

9 Schiehlen, W. O., 1986. Technische Dynamik. Teubner. Stuttgart.

10 Papastavridis, J. P., 1990, "Maggi's Equations of Motion and the De. termination of Constraunt Reactions," AIAA Journal of Guidance, Control, and Dynamics, Vol. 13. pp 213-220.

II Nikravesh. P. E., 1990, "Systematic Reduction of Multibody Equations of Motion to a Minimal Set," International Journal of Non-Linear Mechanics. Vol. 25. Pp 143-151.

12 Blajer, W., 1992. "A Projection Method Approach to Constrained Dy. namic Analysis." ASME Journal of Applied Mechanics, Vol 59, pp. 643-649. 13 Walton, W C.. Jr, and Steeves, E C . 1969, "A New Matrix Theorem and Its Application for Establishing Independent Coordinates for Complex Dynamical Systems with Constraints." NASA Technical Report. TR R-326.

14 Manı, N K. Haug. E J, and Atkison, K E, 1985. "Application of Singular Value Decomposition for Analysis of Mechanical System Dynamics," asme Journal of Mechanisms. Transmissions, and automation in Desici, Vol. 107. PP. 82-87.

15 Singh, R P., and Likıns, P. W , 1985, "Singular Value Decompositıon for Constrained Dynamical Systems." ASME Journal of Applied Mechanics. Vol 52, PP. 943-948

$16 \mathrm{~K}$ m. S S , and Vanderploeg. M J . 1987. "QR Decomposition for State Space Representation of Constrained Mechanical Dynamic Systems," ASME Jolinal of Mechanisms. Transmissions, and Attomation in Design. Vol. 108. pp 183-188.

17 Amirouche, F M L , Jia. T , and Ider, S. K., 1988, "A Recursive House. holder Transformation for Complex Dynamical Systems with Constrants," ASME Journal of Applied Mechanics, Vol 55. pp. 729-734

18 Liang. C. G., and Lance, G M.. 1987, "A Differentiable Null Space Method for Constraned Dynamic Analysis," ASME Journal of MEChanisus. Transmissions, AND AUtomation in Desion, Vol. 109. pp. 405-411.

19 Agrawal, O. P., and Sagal, S., 1989, "Dynamic Analysis of Multi-Body Systems Usıng Tangent Coordinates," Compulers \& Siructures, Vol. 31, pp. 349-355

20 Blajer, W , and Parczewski, J . 1989, "On Realization of Program Con. straints Part II-Practical Implications," ASME Journal of Applied Mechanics. Vol 56. pp 680-684.

21 Baumgarte, J., 1972, "Stabilization of Constraints and Integrals of Motion in Dynamical Systems." Computer Methods in Applied Mechanics and Engt. neering. Vol, 1, pp. 1-16.

22 Ostermeyer. G.-P. 1983. "Mechanische Systeme mit beschrankiem Kon. figurationsraum," Ph.D. thesis, Technische Universitat, Braunschweig. FRG.

23 Wittemburs. J., 1977. Dynamics of Systems of Rigid Bodies, Teubner. Stultgart.

24 Huston, R. L.. 1989, "Methods of Analysis of Constrained Multibody Systems," Mechanics of Siructures and Machines, Vol 17, No 2, pp 135-143. 